\section{High-Molecular-Weight Kininogen}

T. Stief

Institut für Laboratoriumsmedizin und Pathobiochemie, Krankenhaus der Philipps-Universität, Marburg, Deutschland

Synonym(e) Williams-Faktor; Fitzgerald-Faktor; HMWK

Englischer Begriff high-molecular-weight kininogen

Definition High-Molecular-Weight Kininogen ist ein Cofaktor für die Aktivierung von > Präkallikrein zu Kallikrein („F12a/Kallikrein Loop“). Kallikrein und FXIIa setzen aus HMWK das vasoaktive Bradykinin frei.

Beschreibung High-Molecular-Weight Kininogen (HMWK) und Low-Molecular-Weight Kininogen (LMWK) sind Produkte des gleichen Gens, das auf dem langen Arm von Chromosom 3 (3q26-qter) liegt. Beide Produkte sind das Ergebnis eines alternativen Splicings. LMWK ist ein $68 \mathrm{kDa}$ großes $\beta$-Globulin mit einer Plasmakonzentration von $90 \mathrm{mg} / \mathrm{L}$ und HMWK ein $120 \mathrm{kDa}$ großes $\alpha$-Globulin mit einer Plasmakonzentration von $80 \mathrm{mg} / \mathrm{L}$. Hepatozyten exprimieren beide Proteine, Endothelzellen nur HMWK. HMWK konnte auch in Thrombozyten, Granulozyten und in renalen Tubuluszellen nachgewiesen werden. HMWK und LMWK sind Multidomänproteine. Domäne 4 enthält die Bradykininsequenz. FXIIa (F12a) und Kallikrein spalten HMWK und setzen vasoaktives Bradykinin frei. Die Domäne 5 von HMWK bindet an negativgeladene Oberflächen (altered matrix), an Neutrophile, Endothelzellen und Thrombozyten. Ein angeborener Mangel (Williams-Faktor-Mangel, Fitzgerald-Faktor-Mangel) führt nicht zu einer erhöhten Blutungsneigung. Ein erworbener Faktorenmangel findet sich beim erhöhten Verbrauch von Kontaktfaktoren (Sepsis, Polytrauma, disseminierte intravaskuläre Gerinnung (DIC)) oder bei schweren Leberfunktionseinschränkungen. Ein HMWK-Mangel kann mit einstufiger aPTT gemessen werden.

\section{Literatur}

Colman RW (2001) Contact activation pathway: inflammatory, fibrinolytic, anticoagulant, antiadhesive, and antiangiogenic activities. In: Colman RW, Hirsh J, Marder VJ et al (Hrsg) Hemostasis and thrombosis. Lippincott Williams \& Wilkins, Philadelphia, S 103-121

Stief TW (2012) $\mathrm{Zn}^{2+}$, hexane, valproate, or glucose in two purified systems of F12-PK-HMWK. Hemost Lab 5:35-50

Stief TW, Mohrez M (2012) HMWK increases or decreases F12a generation dependent on the contact trigger concentration in two purified systems. Hemost Lab 5:51-65 